\title{
The Impact of Technology on Adolescent Behavior
}

\author{
PhD. Monika Cenameri ${ }^{1}$ \\ Poleta Taçi (Luga) ${ }^{2}$ \\ ${ }^{1}$ Department of Social Work and Social Policy, Faculty of Social Sciences, University of Tirana \\ IInfectious Diseases Specialist 'UHC' Mother Tereza, Infectious Diseases Service \\ Email: monikacenameri@yahoo.com
}

Doi:10.5901/ajis.2015.v4n2s2p288

\begin{abstract}
Information and communication technologies have become the determining feature of our time, making obviously our lives different from what they were once ago. The Internet is without a doubt very popular among teenagers, but as radio, movies, television were initially treated suspiciously and are seen as "corruption" to the youth, also the internet is seen with skepticism for its negative impact on the youth. Adolescents have the highest level of Internet use compare to other groups. They are inclined to stay awake until late at night connected to the internet behind a screen, ignoring social interaction, homework, school, and the world in general. This kind of adolescent's behavior has become the concern of parents but not only because their behavior is assuming the dimensions of an internet addiction towards technology. The aim of the study was to assess the effects of technology on adolescent's behavior. This is a transversal study, which through quantitative methods of data collection describes and analyzes simultaneously. In this study have participated 810 adolescents $(N=810)$ high school students belonging to 14 schools of the city of Tirana. There has been applied two -stages cluster sample and the formula used to calculate the sample size was standard. Results showed that adolescent's internet use is not just a virtual environment or parallel but an integral part of their lives. Adolescences existing behavior is being redefined into the global reach of digital technologies and the growing concern of parents about the impact of technology on adolescent's behavior is real. For example, it was demonstrated in all correlations between the time that adolescents stay in the Internet and the impact that it has on their behavior, which were conformed strongly in all values (chi square ranged from 300 to 550, and the p value was always 0.0000 ).
\end{abstract}

Keywords: Adolescence, internet access, internet addiction, emotional cost, virtual communities.

\section{Introduction}

Contemporary society is in a massive process of change, called digitalization. A part of the communication models and existing behavior is redefined, they are reintegrated in a technological approach with new content, adapted to the contemporary profile of a changing society. Information and communication technology has overcome geographical boundaries and territories, giving life a new dynamic. Extension and expansion of the use of technologies after 1970, as television, electronic mail, mobile phones and optical cables significantly influenced the way we communicate. After 1990, with the invention of the web, began to generate a new space called virtual space or virtual world, which reached in 2008 about 48 billion web pages. The beginning of this millennium marked the growth of computer networks (internet use), making possible the connection of people worldwide without any social, gender, cultural, economic, time and racial distinction. Europe is the region with the highest level of internet spread in the world (75\%), in second place is USA with 61\%. (ITU).

The structure of internet is radically different from centralized systems or partly "decentralized". But internet is a network "distributed" equally, in which each node can be connected to any other node to choose, as appropriate, in a suitable way. This particular special construction structure makes it different, which means it does not have a central point. Each of its "infinite" nodes may be its center.

So, does not exist any "gentleman named internet", to dictate or to control opinions, behaviors or attitudes on the network. Internet in Albania appeared for the first time in 1995 by the office for United Nations Development Program (UNDP) in Tirana, which launched the internet project (Internet free of charge to Universities and Academic Institutions). A year later, for the first time in Albania began mobile service based on the GSM standard, provided by AMC. This service until 1999 was available only in area of Tirana-Durres, in western lowland and numbered about 15 thousand users. 
In 1997 internet delivery initiative was supported by the SOROS Foundation, which built its internet network aimed at providing this service free of charge to state institutions and Non-governmental organizations. Because of legal obstructions, this service was not offered on a commercial base up to 1998. Currently in Albania the number of internet users has reached 1.44 million (ITU). Internet use in Albania during years, in percentage is presented as below:

\begin{tabular}{|l|l|l|l|l|l|l|l|l|l|l|l|l|}
\hline 2000 & 2001 & 2002 & 2003 & 2004 & 2005 & 2006 & 2007 & 2008 & 2009 & 2010 & 2011 & 2012 \\
\hline 0.11 & 0.33 & 0.39 & 0.97 & 2.42 & 6.04 & 9.61 & 15.04 & 23.86 & 41.20 & 45.00 & 49.00 & 54.66 \\
\hline
\end{tabular}

Albania is among the most passionate internet users in Europe, because we have achieved the second place in the world for the growth in this area in the period 2006 - 2010. Albanians use mobile phone a lot, compared with rates of usage in Europe in reference of the 2011 census. Resident population in Albania was 2,821,977 people, meanwhile the number of subscribers who had a mobile phone, according to the same source was 4,547,807 thousand. If you do a simple calculation, almost all Albanians have had in 2011 two mobile phones meantime are remained (1,725,830 phones). According to AKEP (2012) resulted that the number of families that have access to broadband internet in Albania is about 161 thousand, or approximately $22 \%$ of families. Based on these data, the number of broadband access (fixed and $3 \mathrm{G}$ USB / modem) for 100 inhabitants is around 6.2\%, compared to 3.7\% that was in late 2010.

Naturally, that this rapid spread of internet has its impact on the entire population, but especially among young people due to the characteristics that has this target group. In Albania there are no statistics to show how exactly the number of teenagers who use internet is. This deficiency is not only felt in official statistics, but it is also felt on statistics provided by the companies that provide internet service in the country.

The problem became deeper even more by the fact of not knowing the ways in which Albanian teenagers use the internet. This age is called delicate age, because the problems that teens faced combine and integrate in the physical, physiological, psychological and social aspect. In the period of transition from childhood to youth, the child begins to recognize his/her opportunities and skills, confronts himself/herself with others, aims to achieve higher levels of social status, wants to take a larger volume of information, wants to know the world more, wants his/her voice to be felt more around, feels that he/she "knows" and he/she is "something" and requires affirmation from adults about this "something", understands that is not a child, but however he/she cannot be an adult.

Recognizing the effect of internet in Albanian teenager's life is turned to an immediate need today, taking into account two contradictory characteristic of the internet as a medium of individual versus an interactive environment. Technologies in themselves are real asset and with their impact are able to devise new forms of communication and behavior among individuals as well as the diverse approaches in the relations of individuals with the world around them. In the twentieth-century, mainly the youth contact with others through communication face-to-face and by telephone. Nowadays, teenagers that are users of the internet, use the computer as the most appropriate tool to stay in touch with others.

Because of these implications and consequences that they bring to the behavior of adolescents, their study and analysis is a necessity. In this context, this study aims to identify and analyze the level of involvement of Albanian teenagers in the virtual world, and its impact on their behavior, presenting opportunities, implications, challenges and risks that come from the inclusion in the virtual world.

\section{Research Methodology}

This is a transversal study, which through quantitative methods of data collection, describes and analyzes the use of internet and social networks of Albanian adolescents and the impact that they have on their behavior.

\subsection{Study Population}

Although the use of internet in recent years expressed a very fast growth dynamics in the entire country according to official statistics, Tirana has the greatest spread in using this technology (2011). 


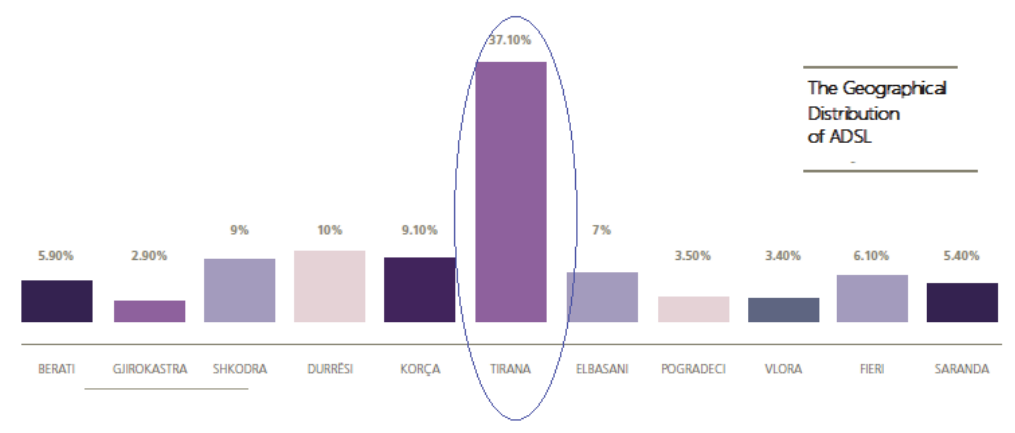

On the other hand adolescents are among the most diligent users of this technology. This study is centered on the students of 14 high schools in Tirana, which had a total number of 15,217 adolescents. In this population are not included the students of professional and private schools. Defining the target population of this study was realized based on data from Tirana Regional Education Directorate.

\subsection{Sample}

In this study is applied the sampling with clusters at two levels (two-stage cluster sample). In this case cluster represents a group of the population, which may have similar characteristics related to the objective of the study, and constitutes the sampling unit (instead of the individual, which is in the case of simple random sample). In our sample of more than fifteen thousand individuals, when the expected percentage of internet use can be assumed $50 \%$ ( since there are no previous studies carried out in this area ), and a study power of $95 \%$ and precision of results $\pm 3.3 \%$, sampling was calculated by the formula:

$$
n=\frac{Z^{2} p(1-p)}{d^{2}}
$$

This sample was about $\mathbf{8 1 0}$ teens. Completion of the questionnaires from randomly selected individuals in each cluster had a zero level of rejection by students.

\section{Results and discussion}

\subsection{The use of internet and social networks by teens}

In this study participated $\mathrm{N}=810$ adolescent high school students belonging to the age group $14-18$ years. $98.9 \%$ of adolescents are internet users and $94.7 \%$ are users of social networks. This high percentage of internet use among adolescents is not statistically significant $(p=0.06)$, in determination of the internet use by adolescents according to their gender. On the other hand the use of social networks has a slightly lower percentage than the use of internet. Boys use social networks more than girls and this percentage varies from $98 \%$ for boys and $93 \%$ for girls. The high percentage of internet users is indicative of the large spread of internet in our country in recent years. The inclusion in the virtual world of adolescent's part of this study, beginnings earlier than pre- adolescence (11-14 years). In cumulative percentage Albanian teenagers, in $44.1 \%$ of cases, have started using the internet at the age of eleven. In the virtual space of social network, an alarm indicator is the average age of starting using of social networks by Albanian teenagers, which in adolescent's part of the study was 12.3 years, when the age to be registered in a social network, such as "Facebook" or "Messenger" is over 13 years old.

The start of using the internet by adolescents in the study in $59 \%$ of cases is associated with learning autodidact, without a guardian, who will be able to help the teens navigate in internet. This way of learning, which at first glance does not seem to present any interest, but when it comes to internet we do not always feel comfortable because there may be a street or "paths" of navigation where we all would not like to pass our children. Referring ecological view this kind of experience demonstrates the development of adolescents as well as their capacity to discover this environment and to contribute to it. Technological environment becomes part of the "microsystem" of adolescents, drawing their attention through technological devices. 


\subsection{Amount of time spent online}

Another important indicator in internet usage in adolescence is the time spent on the internet and social networks and frequency of their use. This time serves as an indicator in the involvement of the virtual world in the lives of adolescents. The percentages show that the mostly time spend by Albanian teenagers on the internet, belong to the range of 3-4 hours per day by (42\%) followed by the interval 5-6 hours a day by (27\%) of the adolescents. Regarding to the highest frequency of social networks usage, in each entry a day on the frequency (more than 2 hours) represents 62\% of the sample in the study, exactly 477 teenagers. The other percentage varies from:

- $1-2$ hours $21 \%$

- $31-60$ minutes $12 \%$

- $11-31$ minutes $3 \%$

- 10 minutes $2 \%$

Learning how to balance the time spent on the internet with all other life activities is one of the biggest challenges of the digital age. Children spend more time in their engagement in the virtual world. The virtual world is full of interesting and fun activities designed especially to "bound people after it" and to draw the attention of adolescents. Interference between social space, virtual space and technology space generate new behaviors, especially among the adolescents, and whether to refer (Marcia) in presence and/or absence of basic dimensions of exploration and engagement, they provide various forms of identity or statuses identity (Marcia 1976).

\subsection{Emotional cost of internet use by adolescents}

Dependence of children and adolescents on internet is a growing concern worldwide. The internetnavigation is a vital part of the modern world, although the internet has become an important educational tool for adolescents, as mentioned above. In addition is stressed the fact that it serves as a tool to pass the time, to have fun and interact with others. However, these citations become the reason for an escape or attration on the internet by many children and adolescents. The internet gives you the opportunity to communicate and chat with anyone, and play exciting games or challenging games against other players in every corner of the globe, with a click of the "mouse" you can enter a different world, where real life problems disappear providentially and are not present anymore, where all the things that someone wants or seeks to prove may become possible.

Just as addition to drug and alcohol, internet offeres children and teenagers other ways of escaping from painful feelings or worrying situations. They sacrifice the hours needed for sleep to spend time on internet, and teens go away from family and friends to go in a more comfortable world called the virtual one. Adolescents in the study $(\mathrm{N}=810)$ have confirmed this fact with their answers where data show that the alternatives "always", "often" and "sometimes" are in high percentage of some of the feelings, perceptions and not positive experiences being experienced from them as a result of using internet. These experiences and perceptions affect the adolescent's behaviour, becoming influential factors in their behaviour.

- $\quad$ In $45 \%$ of cases adolescents said they "often" lost sleep as a result of using internet. Some others studies have reached the same conclusion which emphasize the concerns for the welfare of adolescents, which is related to the little time that adolescents spend sleeping.

- $\quad$ In $43 \%$ of cases adolescents expressed that "always" have emotional explosion as a result of internet use, while their age is an age which presents contrasts due to the transition from one emocional condition to another. In these circumstances emotional explosions take unimagined shapes and sizes.

- In $42 \%$ of cases what happens "often" to teens is losing the sense of time on internet. It is this fact, which leads to a chain reaction that is followed by the complaints of parents for time spent on-line by their children. Parents that were part of the questionnaire in $64 \%$ of cases said that their children "always" and "often" lose the sense of time when they are on-line, in $50 \%$ of cases they "often" and "sometimes" neglect their friends to stay on-line, in $50 \%$ of cases they "often" and "sometimes" get low marks because they want to stay on internet, in 31\% of cases they "often" lie their parents for the amount of time they spent on internet, in 59\% of cases they "often" and "sometimes" use the internet only to kill time without a specific reason, in $40 \%$ of cases they feel anxious after using the internet, etc.

The effects caused by the virtual world in adolescents behavior were analyzed with time (hours) of staying online of the teens that were part of our study. This correlation was confirmed strongly in all experiences and behaviors (chi square ranged from 300 to 550, while the value of $p$ was always 0.0000 ). These data demonstrate WHY the internet is called 
"the technology of loneliness". In such cases internet becomes a factor to separate teenagers from communicating with other people (family, relatives, friends, peers) by isolating them in social and emotional aspects and depriving them from face-to- face communication.

Studies show that the situation is more problematic for children and adolescents who do not have a proper education or do not have the skills to create social relationships. They can develop inadequate or excessive habits because of internet, they feel lonely and have trouble to make new friends. In such conditions they make friendship with invisible strangers that they have recognized on the internet space, in search of attention and missing society in their real lives. Teens that come from families with significant problems can use the internet to avoid problems. They may receive instant messages instead of social relations, affecting in the way they interacted with their peers.

\subsection{The forms of communication used by teenagers}

The acceptance by peers and contacts with them are important elements of adolescent's life. The intensity of the virtual world is a factor that had forced teenagers to choose alternative forms of communication, different from those used once. These new forms of communication, apparently faster have influenced the behavior of adolescents, making them less communicative in the real world, and affecting at the same time cognitive, emotional and behavioral aspects. From the analysis of the communication that occurs more frequently among adolescents in the study with their friends (when they are near each others) was founded that the most common communication occurs "face to face" in 81\% of cases, in $13 \%$ of cases occurs "sending messages" and in $6 \%$ of cases occurs "spoken on the phone". The activity used less by adolescents with their friends (when they are close to each-others) is speaking on the phone. This behavior can have its arguments, which lead to a hypothesis to justify the fact that adolescents choose to use more messages than calling by mobile phones. Teenagers send messages because they are more concise (short), faster and with less spending. (it remains to be tested in other studies). Interestingly is the comparison of the forms of communication that teenagers use and the time they spend online. The less time teenagers spend online, the more time they dedicate to communication "face- to-face ". This type of communication has recorded the highest percentage (88 \%) of time to those adolescents who stay online a few minutes a day. Meanwhile the lowest percentage (70 \%) was recorded in adolescents who spend more than 9 hours online. This correlation that was important part of this study was statistically significant and showed that the amount of time that young people spend on internet is an important factor in cultivating new ways of communication that send them away from real communication even when they have their friends close.

Also, messaging and talking on the phone, if we refer to the data of the study, are related to the amount of time spent on internet and present a declining tendency in the reduction of hours that teens stay on internet. The amount of time spent on internet is an important factor that affects in many aspects of adolescent behavior.

This conclusion that has also resulted in some different studies, such as: (Kraut st al.s.1998), which found that excessive use of internet is accompanied by reduction of communication in family as well as reduction of social circle, at the same time with the increase of loneliness and depression. Nie st al. (2002) stressed that the use of internet affects the displacement of social time. Based on daily time data they found that excessive internet use is associated with limitation of the time devoted to family and society. The study also analyzed the forms of communication that adolescents choose to use with their friends (when they are not close to them) from which resulted that the highest percentage $47 \%$ of the adolescents choose to chat with friends (when they are not close to them), $25 \%$ of adolescents talk on the phone with friends (when they are not close to them), $23 \%$ of them send messages and $5 \%$ of them send e-mails. By the correlation of hours spent on internet and communication by phone of adolescents with their friends (when they are not close to them), it was found that the growth of hours spent online is followed by lower percentage of communication by phone of teenagers with their friends, for example, adolescents who stay for more than 9 hours online, only in $10 \%$ of cases use the phone to communicate, on the other hand teens who spend a few minutes a day on internet in $38 \%$ of cases communicate by phone.

\subsection{The roles that internet plays in adolescents lives}

Different from the television in which the viewer is passive and has a small control when changing channels, the use of computer is very active, giving to the user a considerable control. Seen in this perspective adolescents have said that internet plays several roles in their lives. Adolescents in the study have grouped these roles as follows:

- $\quad$ The role of internet in information (in frequencies always \& often) $=84.9 \%$

- The role of internet in fun (in frequencies always \& often) $=74.2 \%$ 
- The role of internet in the detachment from reality (in frequencies always \& often) $=60.3 \%$

- The role of internet in the creation of social connections (in frequencies always \& often) $=55.3 \%$

- $\quad$ The role of internet in creating a social identity (in frequencies always \& often) $=27.7 \%$

All these roles are attributed to the internet by adolescents, while some of them have positive effects and some others not. In $60.3 \%$ of cases adolescents have said that the internet plays a role in their detachment from reality. Some studies noted that social interaction is a worthwhile activity that has been replaced by the use of the internet (Kraut, Kiesler, Mukopadhyay \& Scherlis 1998; MESCH 2003; Nie, Hillygus \& Erbring 2002). One of the studies mentioned above (Kraut et al.'s 1998) emphasis the replacement of social interaction time with the use of the internet. This study, called "HomeNet" explores the relationship between internet use, social involvement and psychological consequences.

From this study is found that great use of internet is accompanied with: a decrease of communication in family, a reduction of the size of the local social circle and the increase of loneliness and depression. The research of (Mesch 2003, 2006), reviewed that the use of internet among Israelite youth was negatively related with family closeness and positively with family conflicts. Also, the study of (Nie et.al 2002) stressed that the use of internet affects in displacement of social time.

\section{Conclusion}

Various studies show that for today's youth the internet is not just a virtual environment or parallel, but an integral part of their real lives. Internet as an integral part of adolescent life does not exclude Albanian teenagers. Teenagers belong to the generation of "digital born". While teens are studying, they chat on, listen to music, send and receive messages, watch videos in YouTube. Nowadays teenagers are the most loyal internet users.

Sociologists and philosophers of network affirm that "the internet infrastructure can be considered equivalent with the one of a biological system and of an ecosystem". Viewed in this perspective internet must be evaluated not as a technology but as a culture and when we talk about culture, it cannot be formed in a short time and it must pass through a process of evolution.

Internet is really an "ocean of opportunity", but it must be consumed in a moderate and balanced way and all this depends on the culture and the behavior of people.

To apply in a positive way the full potential offered by the virtual world is important to teach the adolescents with responsible and ethical habits, because in today's world social changes are judged as a result of technological changes. What we must do is to teach them:

- To use online time wisely.

- To balance the time spent on internet with all activities as one of the major challenges of the digital age.

- To understand what information is safe to share online and what is not.

- To preserve the digital reputation.

- To understand that everything on internet is sustainable, replicable and can be seen by wide invisible audiences.

- To grow up creating and maintaining safe and healthy relationships.

Adolescent problems with the virtual world have to be seen in family and school dimensions and in professional's level too.

\section{References}

AKEP. (2013) Statistical indicators of electronic communications market (2013)

Arnett, J. J. (2004). Adolescence and Emerging adulthood: A Cultural Approach (2nd ed.). Upper Saddle River, NJ: Pearson Prentice Hall.

Bronfenbrenner, U., \& Morris, PA (1998) London: Maximillian Ecology of DEVELOPMENTAL processes.we. In W. Damon (Series Ed.) \& RM Lerner (Vol. Ed.), Handbook of Child Psychology: Vol. 1: Theoretical Models of Human Development (pp. 993-1028). New York: Wiley.

Cummings, J., Butler, B., \& Kraut, J. (2002). The quality of online social relationships. Communications of the ACM, 45, 103-108.

Cross-Cutting Strategy for Information Society 2008-2013

EASTIN, MS, Greenberg, BS, \& Hofschire, L. (2006). Parenting the Internet. Journal of Communication, 56, 486-504.

EU Kids Online deliverable D4: Core fi ndings to the European Commission Safer Internet Programme (13 January 2011). Risks and safety on the internet: The perspective of European Children. Full fi ndings and policy implications from the EU Kids Online survey of 9-16 year olds and theire Parents in 25 Countries.

Maigret, E. (2010) La sociologie de la communication et des medias. 
MESCH, G. S. (2003). The family and the Internet: The Israeli case. Social Science Quarterly, 84, 1039-1050.

MESCH, G. S. (2006). Family Characteristics and intergenerational confl ICTS over the Internet. Information, Communication and Society, 9, 473-495.

N., Hillygus, D., \& Erbring, L. (2002). Internet use, interpersonal relations, and sociability. In Haythornthwaite B. Wellman \& C. (Eds.), The Internet in everyday

Kraut, R., Mukhopadhyay, T., Szczypula, J., Kiesler, S., \& Scherlis, W. (1998) .Communication and information: Alternative uses of the Internet in households. In Proceedings of the CHI 98 (pp. 368-383). New York: ACM

Kraut, R., Patterson, M., Lundmark, V., Kiesler, S., Mukopadhyay, T., \& Scherlis, W. (1998). Internet paradox: A social technology That reduces social involvement and Psychological Well-Being? American Psychologist, 53, 1017-1031.

Strategies for the Promotion of Broadband Services and infrastructure: a case study on Albania September 2012

Thomollar, D. (2012) Internet and society. 